\title{
Maternal and child patterns of Medicaid retention: a prospective cohort study
}

\author{
Susmita Pati ${ }^{{ }^{*}}$ (D), Rose Calixte ${ }^{1}$, Angie Wong ${ }^{1}$, Jiayu Huang ${ }^{1}$, Zeinab Baba ${ }^{2}$, Xianqun Luan ${ }^{3}$ and Avital Cnaan ${ }^{4,5}$
}

\begin{abstract}
Background: We sought to determine whether maternal Medicaid retention influences child Medicaid retention because caregivers play a critical role in assuring children's health access.

Methods: We conducted a longitudinal prospective cohort study of a convenience sample of 604 Medicaid-eligible mother-child dyads followed from the infant's birth through 24 months of age with parent surveys. Individual enrollment status was abstracted from administrative Medicaid eligibility files. Generalized estimating equations quantified the effect of maternal Medicaid enrollment status on child Medicaid retention, adjusting for relevant covariates. Because varying lengths of gaps may have different effects on child health outcomes, Medicaid enrollment status was further categorized by length of gap: any gap, $>14$-days, and $>60$-days.

Results: This cohort consists primarily of African-American (94\%), unmarried mothers (88\%), with a mean age of 23.2 years. In multivariable analysis, children whose mothers experienced any gaps in coverage had 12.6 times greater odds of experiencing gaps when compared to children whose mothers were continuously enrolled. Use of varying thresholds to define coverage gaps resulted in similar odds ratios (>14-day gap $=$ 11.8 , >60-day gap =16.8). Cash assistance receipt and maternal knowledge of differences between Temporary Assistance to Needy Families and Medicaid eligibility criteria demonstrated strong protective effects against child Medicaid disenrollment.

Conclusions: Medicaid disenrollment remains a significant policy problem and maternal Medicaid retention patterns show strong effects on child Medicaid retention. Policymakers need to invest in effective outreach strategies, including family-friendly application processes, to reduce enrollment barriers so that all eligible families can take advantage of these coverage opportunities.
\end{abstract}

Keywords: Medicaid retention, Health insurance, Children

\section{Background}

Children with health insurance coverage gaps are less likely than those with continuous coverage to have access to a regular source of care for routine preventive needs (e.g., well-child care visits, developmental screening, immunizations) $[1,2]$. This phenomenon contributes to poor health outcomes [1, 2]. In fact, children with brief health insurance coverage gaps have comparable health outcomes to children who are continuously uninsured $[3,4]$. In recent years, many states have simplified enrollment and renewal procedures for public insurance programs to reduce the number of eligible children losing coverage for procedural reasons $[5,6]$.

\footnotetext{
* Correspondence: susmita.pati@stonybrook.edu

${ }^{1}$ Division of Primary Care Pediatrics, State University of New York at Stony

Brook, 100 Nicolls Rd, Stony Brook, NY 11794, USA

Full list of author information is available at the end of the article
}

However, coverage gaps affected as many as $33-40 \%$ of children transitioning from Medicaid-based public insurance plans to separate Children's Health Insurance Program public insurance plans [7].

Individual characteristics and policy-level factors are known to influence child Medicaid retention. Our work in this study is theoretically grounded in Anderson and Aday's widely used framework for studying access to care that highlights the interaction between the organization of health care services and individual characteristics that affect access to care [8]. For instance, Hispanic children and older children are disproportionately more likely than their peers to experience coverage gaps [4,9-11]. At the policy level, the 1997 passage of welfare reform that

(C) The Author(s). 2018 Open Access This article is distributed under the terms of the Creative Commons Attribution 4.0 International License (http://creativecommons.org/licenses/by/4.0/), which permits unrestricted use, distribution, and 
separated cash assistance (i.e. Temporary Assistance to Needy Families [TANF]) and Medicaid eligibility resulted in significant confusion about eligibility and application processes that, in turn, resulted in significant drops in enrollment in both programs [12, 13]. In addition, one recent study revealed that with only one exception all state Medicaid renewal applications in 2008 were written at the fifth grade level or higher, suggesting that poor caregiver literacy may adversely affect child Medicaid retention [14]. Several studies have associated parental health insurance status with that of their children, but did not include individual-level information about parental health literacy or TANF eligibility [15-17]. Though children rely on caregivers to initiate enrollment and complete renewals, the direct longitudinal influence of maternal Medicaid enrollment status on child Medicaid retention has not been well quantified in population-based studies.

The primary hypothesis of this study was that maternal Medicaid disenrollment increases the likelihood of child Medicaid disenrollment. We also explored various thresholds for defining coverage gaps and quantified the time to the child's first disenrollment to better understand this relationship. Our secondary goal was to advance our understanding of the influence of other plausible factors on child Medicaid retention that have not been fully explored to date. These factors include maternal health literacy, cash assistance receipt, and maternal knowledge about the separation of eligibility determinations for TANF and Medicaid. In this study, we focused on the association between maternal and child disenrollment, for any reason, because this issue is critical from the perspectives of patients and providers.

\section{Methods}

\section{Study design, study population and data sources}

We performed a prospective cohort study of mother-infant dyads enrolled in the Health Insurance Improvement Project (HIP). The overarching aim of the HIP study was to identify individual characteristics and policy factors that influence child Medicaid retention. This study was approved by and carried out in accordance with guidelines from the Institutional Review Boards at the University of Pennsylvania, The Children's Hospital of Philadelphia, and Stony Brook University. Between June 2005 and August 2006, study subjects who were enrolled or eligible for Medicaid as indicated in the hospital medical record were recruited as a convenience sample from the post-partum wards of a large urban hospital shortly after the infant's birth. As previously published, we enrolled 744 of the 1395 eligible mother-child dyads (Figure 2 in Appendix 1) [18]. If multiple children (e.g., twins) were born to the same mother, only one child was chosen randomly to be included in the study. Upon enrollment, mothers completed a baseline survey, which included socio-demographic information and the Short-Test of Functional Health Literacy in Adults (S-TOFHLA) [19, 20]. Subsequently, a computer-assisted survey instrument was administered via telephone every 6 months through age 24 months by trained staff to collect data about additional covariates. We obtained informed consent from all subjects in accordance with guidelines from the Institutional Review Boards at the aforementioned institutions.

\section{Measures}

Our primary predictor of interest was maternal Medicaid disenrollment and the primary outcome of interest was child Medicaid disenrollment. We linked administrative Medicaid eligibility data (including category of eligibility as well as enrollment and termination dates) for mothers and children using individual identifiers collected at enrollment. We defined the start of each subject's observation period as the child's date of birth and the end as 6 months after the last follow-up survey administered. We assumed all subjects had Medicaid coverage from birth through the end of the observation period except for those with a Medicaid termination date in the eligibility data occurring earlier than the end of the observation period. We censored observations on subjects who reported moving out of state, entering foster care or adoption services, at the time point of the event. Of the 744 enrolled dyads, we successfully linked 604 (81.2\%) to administrative Medicaid eligibility files and this group comprised the analytic study sample. Notably, matched mother-child dyads were more likely to be U.S. born than unmatched subjects (Table 6 in Appendix 2).

We defined disenrollment as any period without Medicaid coverage at any time during the observation period. Notably, there were 48 infants who were not enrolled in Medicaid at birth (mean number of days to first enrollment after birth: 84.7, standard deviation 139.7). For these infants, we considered this gap between birth and first enrollment their first disenrollment. We used three different thresholds to define uninsured periods for the child: any gap (i.e. any period without coverage), $>14$ days gap, and $>60$ days gap. We selected the 14 day threshold because the American Academy of Pediatrics recommends that newborns have three health supervision visits in the first 2 weeks of life [21] and we generalized this threshold to all ages because any gap in coverage for young children may adversely affect health care access and, in turn, outcomes. We selected the 60 day threshold because Medicaid agencies can take up to 45 days to process an application [22]. We applied the 'any gap' definition to maternal Medicaid coverage data in order to keep the definition of maternal disenrollment consistent across models. We did not classify switches from one eligibility category to another while maintaining coverage as a disenrollment event. For purposes of determining enrollment trends, we recorded any change in enrollment status 
(e.g., disenrollment, re-enrollment) in either the mother or the child as a unique period. Notably, each subject could have multiple enrollment periods (e.g., enrolled April 2006-September 2006, disenrolled October 2006-November 2006, re-enrolled December 2006-March 2007, etc.). For each period, we classified Medicaid eligibility categories as cash-assistance (i.e. TANF or Supplemental Security Income) related or not.

\section{Covariates}

We collected covariates known to influence Medicaid and/or public program participation $[10,12,23-26]$ in the following ways. We collected socio-demographic information using items adapted from the National Health Interview Survey administered at birth in-person and then every 6 months via telephone for the remainder of the study period [27]; maternal health literacy (using S-TOFHLA) [19, 20] and maternal knowledge that Medicaid and TANF have different eligibility criteria were collected in person at enrollment. We assessed maternal instrumental and relational social support using scores from the Maternal Social Support Index (MSSI) that was administered at 12, 18, 24 months after enrollment; a higher score indicates greater social support [28].

\section{Statistical analyses}

The main goal of our analyses was to assess whether maternal Medicaid disenrollment was significantly associated with child Medicaid disenrollment status after adjusting for relevant covariates. We included all covariates except maternal age (continuous) as categorical variables. We treated maternal health literacy and maternal knowledge that Medicaid and TANF have different eligibility as fixed covariates. Maternal health status, employment status, social support, household income, and housing situation changed over time and were included as time varying covariates. We treated all other factors as fixed covariates using values obtained at the 6-month survey consistent with the observed patterns in the data. When child disenrollment occurred, we used the most recent covariate and mother disenrollment data.

We used generalized estimating equations (GEE) to determine how well child Medicaid enrollment status could be explained by maternal Medicaid enrollment status and the covariates. In the GEE, the child was the cluster and the cluster had as many observations as there were enrollment or disenrollment periods. Thus, a child who was continuously enrolled in Medicaid had one observation in the cluster. If a child disenrolled once and never reenrolled, that child had two observations in the cluster. We calculated odds ratios (ORs) for child disenrollment based on the GEE models to assess the impact of each covariate. We performed sensitivity analyses to test whether the definition of gap (any, > 14 days, > 60 days) affects maternal and child Medicaid enrollment status. We used a best subsets approach to create and choose the best fitting models in order to obtain the most parsimonious and best fitting model that explains child Medicaid enrollment status [29]. We checked the final models to confirm no collinearity problem was present. We examined model fit using the Quasi-likelihood under Independence Model Criterion (QIC) [30, 31].

We next used the Cox proportional hazards model to determine the response of 'time to child's first Medicaid disenrollment' to maternal Medicaid enrollment status and covariates. Here, we used 'any gap' to define uninsured periods for both child and maternal Medicaid coverage. All changes in time-varying covariates were recorded. We used the most recent covariates and mother disenrollment data when child's disenrollment occurred. We censored a child continuously enrolled in Medicaid during the study period. A child not enrolled in Medicaid on the date of birth had time-to-disenrollment of 0 days. We checked the proportional hazards assumption for each covariate. We calculated hazard ratios for time to child disenrollment based on the Cox proportional hazards model to assess the impact of predictors. We used a best subsets approach to create and choose the best fitting models. There were no collinearity problems in the final models. We examined model fit by Akaike information criterion (AIC).

\section{Missing data}

The fraction of missing survey data ranged from 0.17 to $16.06 \%$ per item, with variables for maternal employment and knowledge that Medicaid and TANF eligibility criteria differ having more than $10 \%$ missing. We performed multiple imputation for missing data using the method of chained equations [32]. To avoid potential bias and potential reduction in statistical power from using only complete observations. All reported results, including standard errors, are from completed datasets using the imputation procedures.

A Type I error level of 0.05 was used for all analyses, and all significance tests were two-sided. SAS $9.3^{\circ}$ was used for analyses.

\section{Results}

The analytic sample cohort consists mostly of young, African-American mothers with more than one child who were not married (Table 1 ). The majority completed high school, had adequate health literacy, and knew that eligibility criteria for TANF and Medicaid differ. More than half of mothers were unemployed or students, did not live in their own housing, and had household incomes of $<\$ 1000$ per month. Among mothers who completed the MSSI $(n=478)$, most mothers reported to having medium to high social support.

Table 1 also shows that the number and proportion of children with gaps in Medicaid coverage changed as the 
Table 1 Population characteristics at child's birth and association with child Medicaid disenrollment

\begin{tabular}{|c|c|c|c|c|c|c|c|c|c|c|}
\hline \multirow[t]{2}{*}{ Characteristics } & \multirow[t]{2}{*}{ N (\%) } & \multicolumn{3}{|l|}{ Any gap } & \multicolumn{3}{|l|}{$>14$ day gap } & \multicolumn{3}{|l|}{$>60$ day gap } \\
\hline & & $\begin{array}{l}\text { Children with } \\
\text { no gap } \\
\mathrm{N}(\%)\end{array}$ & $\begin{array}{l}\text { Children } \\
\text { with gap } \\
\mathrm{N}(\%)\end{array}$ & $P$-value ${ }^{a}$ & $\begin{array}{l}\text { Children with } \\
\text { no gap } \\
N(\%)\end{array}$ & $\begin{array}{l}\text { Children } \\
\text { with gap } \\
N(\%)\end{array}$ & $P$-value & $\begin{array}{l}\text { Children with } \\
\text { no gap } \\
\mathrm{N}(\%)\end{array}$ & $\begin{array}{l}\text { Children } \\
\text { with gap } \\
N(\%)\end{array}$ & $P$-value ${ }^{a}$ \\
\hline Total sample & 604 & $450(75)$ & $154(25)$ & & 469(78) & $135(22)$ & & $502(83)$ & $102(17)$ & \\
\hline \multicolumn{11}{|l|}{ Child gender } \\
\hline Male & $308(51)$ & $224(50)$ & $84(55)$ & 0.30 & $236(50)$ & $72(53)$ & 0.53 & $254(51)$ & $54(53)$ & 0.66 \\
\hline Female & 296(49) & $226(50)$ & $70(45)$ & & $233(50)$ & $63(47)$ & & 248(49) & $48(47)$ & \\
\hline \multicolumn{11}{|l|}{ Maternal age } \\
\hline Under 18 & $54(9)$ & $39(9)$ & $15(10)$ & 0.92 & $40(9)$ & $14(10)$ & 0.74 & $42(8)$ & $12(12)$ & 0.48 \\
\hline $18-24$ & $349(58)$ & $261(58)$ & $88(57)$ & & $274(58)$ & $75(56)$ & & 294(59) & $55(54)$ & \\
\hline 25 and over & 201(33) & $150(33)$ & $51(33)$ & & 155(33) & $46(34)$ & & $166(33)$ & $35(34)$ & \\
\hline \multicolumn{11}{|l|}{ Maternal race } \\
\hline African American & $569(94)$ & $427(95)$ & $142(92)$ & 0.21 & $444(95)$ & $125(93)$ & 0.36 & $475(95)$ & $94(92)$ & 0.33 \\
\hline Other $^{b}$ & $35(6)$ & $23(5)$ & $12(8)$ & & $25(5)$ & $10(7)$ & & $27(5)$ & $8(8)$ & \\
\hline \multicolumn{11}{|l|}{ Maternal education } \\
\hline Less than High School & 198(33) & $153(34)$ & $45(29)$ & 0.51 & 159(34) & $39(29)$ & 0.54 & $168(33)$ & $30(29)$ & 0.69 \\
\hline High School & $147(24)$ & $109(24)$ & $38(25)$ & & $113(24)$ & $34(25)$ & & $122(24)$ & $25(25)$ & \\
\hline More than High School & $259(43)$ & $188(42)$ & $71(46)$ & & 197(42) & $62(46)$ & & $212(42)$ & $47(46)$ & \\
\hline \multicolumn{11}{|l|}{ Maternal health literacy* } \\
\hline Inadequate & $56(9)$ & $38(8)$ & $19(12)$ & $<.0001$ & $41(9)$ & $16(12)$ & 0.0021 & $46(9)$ & $11(11)$ & 0.05 \\
\hline Marginal & $80(13)$ & $58(13)$ & $21(14)$ & & $61(13)$ & $19(14)$ & & $69(14)$ & $11(11)$ & \\
\hline Adequate & $467(77)$ & $353(78)$ & $115(75)$ & & $367(78)$ & $100(74)$ & & $387(77)$ & $80(78)$ & \\
\hline \multicolumn{11}{|l|}{ Other children in household } \\
\hline None & $228(38)$ & $158(35)$ & $70(45)$ & 0.073 & 169(36) & $59(44)$ & 0.26 & 181(36) & $47(46)$ & 0.062 \\
\hline One & $157(26)$ & $122(27)$ & $35(23)$ & & $126(27)$ & $31(23)$ & & $139(28)$ & 18(18) & \\
\hline Two or more & 219(36) & $170(38)$ & $49(32)$ & & $174(37)$ & $45(33)$ & & 182(36) & $37(36)$ & \\
\hline \multicolumn{11}{|l|}{ Prenatal Care, self-reported* } \\
\hline All/Most of the time & $553(92)$ & $411(91)$ & $142(92)$ & 0.28 & $428(91)$ & $125(93)$ & 0.11 & $458(91)$ & 95(93) & 0.04 \\
\hline Some/None of the time & $51(9)$ & 39(9) & $12(8)$ & & $41(9)$ & $10(7)$ & & $44(9)$ & $7(7)$ & \\
\hline \multicolumn{11}{|l|}{ Maternal self-reported health } \\
\hline Score < 80 (poor health) & $224(37)$ & $163(36)$ & $61(40)$ & 0.45 & 169(36) & $55(41)$ & 0.32 & 181(36) & $43(42)$ & 0.24 \\
\hline Score $\geq 80$ (good health) & $380(63)$ & $287(64)$ & $93(60)$ & & $300(64)$ & $80(60)$ & & $321(64)$ & $59(58)$ & \\
\hline \multicolumn{11}{|c|}{ Maternal knowledge that TANF and Medicaid eligibility criteria are different } \\
\hline Yes & $492(81)$ & $377(84)$ & $115(75)$ & $<.0001$ & $387(83)$ & 105(78) & $<.0001$ & $412(82)$ & $80(78)$ & 0.006 \\
\hline No & $112(19)$ & $73(16)$ & $39(25)$ & & $82(17)$ & $30(22)$ & & $90(18)$ & 22(22) & \\
\hline \multicolumn{11}{|l|}{ Travel time to Medicaid office* } \\
\hline$<30 \min$ & $410(68)$ & $305(68)$ & 105(68) & 0.93 & $320(68)$ & $90(67)$ & 0.73 & $341(68)$ & $69(68)$ & 0.96 \\
\hline$\geq 30 \mathrm{~min}$ & 194(32) & $145(32)$ & $49(32)$ & & 149(32) & $45(33)$ & & $161(32)$ & $33(32)$ & \\
\hline \multicolumn{11}{|l|}{ Household income* } \\
\hline$<\$ 1000 /$ month & $410(68)$ & $312(69)$ & $98(64)$ & $<.0001$ & $325(69)$ & $85(63)$ & $<.0001$ & $350(70)$ & $60(59)$ & $<.0001$ \\
\hline$\$ 1000$ or more/month & 194(32) & $138(31)$ & $56(36)$ & & $145(31)$ & $50(37)$ & & $152(30)$ & $42(41)$ & \\
\hline \multicolumn{11}{|l|}{ Marital status } \\
\hline Single/Divorced/Widowed & $533(88)$ & $404(90)$ & $129(84)$ & 0.045 & $422(90)$ & $111(82)$ & 0.013 & $452(90)$ & $81(79)$ & 0.002 \\
\hline Married & $71(12)$ & $46(10)$ & $25(16)$ & & $47(10)$ & $24(18)$ & & $50(10)$ & $21(21)$ & \\
\hline
\end{tabular}


Table 1 Population characteristics at child's birth and association with child Medicaid disenrollment (Continued)

\begin{tabular}{|c|c|c|c|c|c|c|c|c|c|c|}
\hline \multirow[t]{2}{*}{ Characteristics } & \multirow[t]{2}{*}{$N(\%)$} & \multicolumn{3}{|l|}{ Any gap } & \multicolumn{3}{|l|}{$>14$ day gap } & \multicolumn{3}{|l|}{$>60$ day gap } \\
\hline & & $\begin{array}{l}\text { Children with } \\
\text { no gap } \\
\mathrm{N}(\%)\end{array}$ & $\begin{array}{l}\text { Children } \\
\text { with gap } \\
\mathrm{N}(\%)\end{array}$ & $P$-value ${ }^{a}$ & $\begin{array}{l}\text { Children with } \\
\text { no gap } \\
\mathrm{N}(\%)\end{array}$ & $\begin{array}{l}\text { Children } \\
\text { with gap } \\
\mathrm{N}(\%)\end{array}$ & $P$-value ${ }^{a}$ & $\begin{array}{l}\text { Children with } \\
\text { no gap } \\
\mathrm{N}(\%)\end{array}$ & $\begin{array}{l}\text { Children } \\
\text { with gap } \\
N(\%)\end{array}$ & $P$-value \\
\hline \multicolumn{11}{|l|}{ Maternal employment status* } \\
\hline Student & $152(25)$ & 118(26) & $34(22)$ & $<.0001$ & $123(26)$ & $29(21)$ & $<.0001$ & $127(25)$ & $26(25)$ & 0.0002 \\
\hline Full Time & $245(41)$ & $172(38)$ & $72(47)$ & & 181(39) & $63(47)$ & & 198(39) & $47(46)$ & \\
\hline Unemployed & $207(34)$ & $160(36)$ & $48(31)$ & & $164(35)$ & $43(32)$ & & $177(35)$ & $30(29)$ & \\
\hline \multicolumn{11}{|l|}{ Family housing situation } \\
\hline Lives in own housing & $243(40)$ & $234(52)$ & $61(40)$ & 0.890 & $245(52)$ & $51(38)$ & 0.40 & 262(52) & $38(37)$ & 0.30 \\
\hline Rents or lives with relative & $361(60)$ & $216(48)$ & $93(60)$ & & $224(48)$ & $84(62)$ & & $240(48)$ & $64(63)$ & \\
\hline \multicolumn{11}{|l|}{ Maternal social support* } \\
\hline Low & 190(32) & $134(30)$ & $56(36)$ & $<.0001$ & 143(30) & $47(35)$ & $<.0001$ & $157(31)$ & $33(32)$ & $<.0001$ \\
\hline Medium & $135(22)$ & $98(22)$ & $37(24)$ & & $102(22)$ & $33(24)$ & & 108(22) & $27(26)$ & \\
\hline High & $153(25)$ & $102(23)$ & $51(33)$ & & $106(23)$ & $47(35)$ & & $113(23)$ & $40(39)$ & \\
\hline Not collected & $126(21)$ & $116(26)$ & $10(7)$ & & $118(25)$ & $8(6)$ & & $124(25)$ & 2(2) & \\
\hline
\end{tabular}

Notes: Maternal health literacy was assessed using the S-TOFHLA and categorized as inadequate, marginal, or adequate per published technical guidance

(Nurss JR, Parker R, Willams M, Baker D. TOFHLA Test of Functional Health Literacy in Adults. Second ed. Snow Camp, NC: Peppercorn Books \& Press; 2001). Maternal self-reported health was assessed using the SF-36 ${ }^{\oplus}$ (Ware JE, Jr., Sherbourne CD. The MOS 36 -item short-form health survey (SF-36). I. Conceptual framework and item selection. Med. Care. Jun 1992;30(6):473-483). Maternal instrumental and relational social support was assessed using the Maternal Social Support Index and categorized low, medium, or high using tertiles per published technical guidance (Pascoe JM, lalongo NS, Horn WF, Reinhart MA, Perradatto D. The reliability and validity of the maternal social support index. Fam. Med. Jul-Aug 1988;20(4):271-27)

${ }^{a} p$-value is for the $x 2$ test of association

"Includes Hispanic, Asian and "Ethnically-Challenged" (as self-reported)

*Results from 10 imputed datasets. Percentages may not total 100 due to rounding

threshold for defining coverage gaps varied. One-quarter $(n$ =154) of children experienced at least one gap of any length of time in coverage during the first two years of life. Among those with any gap, 135 (22\% of total) children were disenrolled for $>14$ days and 102 (17\% of total) children were disenrolled for $>60$ days. Generally, the characteristics of children with gaps were similar regardless of the threshold used to define coverage gaps. Continuously enrolled children were more likely than children with any gap in coverageregardless of the threshold used to define gaps-- to have single, unemployed mothers with adequate health literacy, who know that Medicaid and TANF eligibility criteria differ, have low household income and have low social support.

Regardless of the length of gap, the distribution of coverage patterns for mothers and children were similar with a greater proportion of mothers experiencing coverage difficulties than children (Fig. 1 Medicaid enrollment patterns and eligibility category for mothers and children, by gap definition, Panel a Medicaid enrollment patterns). Most children and mothers were continuously covered with no change in eligibility category across different gap definitions. When exploring the distribution of Medicaid eligibility categories, specifically cash assistance recipients vs. Medicaid only, we observed that a greater proportion of children than mothers were cash assistance recipients
(Fig. 1 Medicaid enrollment patterns and eligibility category for mothers and children, by gap definition, Panel b Medicaid eligibility category). Comparing mothers and children who received cash assistance, a consistently greater proportion of mothers than children experienced gaps of any length. At the same time, more mothers who were Medicaid-only recipients had coverage gaps than mothers who received cash assistance.

Maternal disenrollment and mother-child cash assistance receipt were strongly and significantly associated with child disenrollment, regardless of the threshold used to define gaps in coverage for children (Table 2). Specifically, maternal disenrollment (defined as any gap in Medicaid coverage based on sensitivity analyses performed) was associated with a more than 10 times increased odds of child disenrollment at all three thresholds for defining gaps. Mother-child cash assistance receipt and child cash assistance receipt had similarly strong protective effects against child disenrollment at all gap levels. Children of married mothers were more likely than others to have gaps $>14$ days or $>60$ days. Maternal lack of knowledge that Medicaid and TANF have different eligibility criteria was associated with increased odds of child having any gap, but not with gaps $>14$ or $>60$ days. Similarly, children whose families were renting or living with friends/relatives were more likely than those living in their own housing to have any gap, but not gaps $>14$ or $>60$ days. 
A

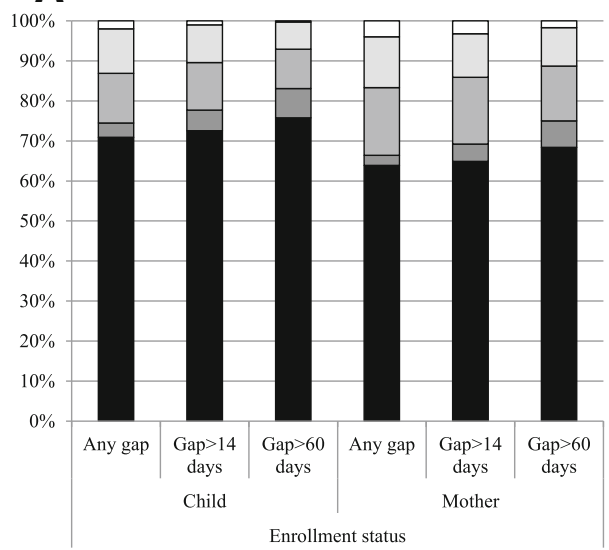

Panel A: Medicaid Enrollment Patterns

$\square$ Two disenrollments

$\square$ One disenrollment

$\square$ Disenrolled prior to end of observation period and never re-enrolled

$\square$ Continuously covered with at least one change in eligibility category

- Continuously covered, no change in eligibility category

\section{B}

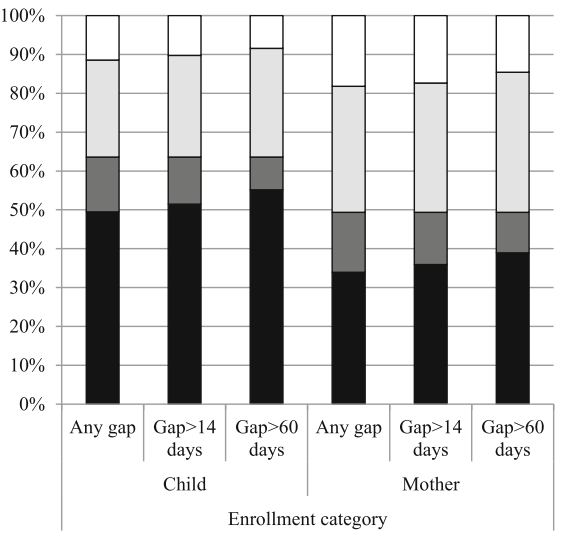

Panel B: Medicaid Eligibility Category

$\square$ Medicaid recipient with gap

$\square$ Medicaid recipient with no gap

$\square$ Cash assistance recipient with gap

- Cash assistance recipient with no gap

Fig. 1 Medicaid enrollment patterns and eligibility category for mothers and children, by gap definition. Panel a Medicaid Enrollment Patterns. Panel $\mathbf{b}$ Medicaid Eligibility Category Notes: Disenrollment was defined as any period without Medicaid coverage at any time during the observation period.Results from 10 imputed datasets.

Notably, maternal health literacy was not significantly associated with child disenrollment.

Maternal disenrollment and mother-child cash assistance receipt were also significantly associated with time to child's first Medicaid disenrollment (Table 3). Maternal disenrollment was associated with a more than 5 times increased rate of child disenrollment. Children whose families were renting or living with friends/relatives had larger rate of disenrollment than those living in their own housing. Children living in households without any other children also had increased rate of Medicaid disenrollment.

The main findings from the single variable analyses persisted in the multivariable analyses with a few notable differences. Using the best subsets approach, maternal disenrollment and mother-child dyad cash assistance status, but not maternal health literacy, were included in the final model (Table 4). Though not significant in single variable results, monthly household income was included in the final best subsets models for any gap and $>14$ day gaps because household income is an explicit criterion for Medicaid eligibility. Controlling for relevant covariates, children of mothers who disenrolled from Medicaid had 10 times greater odds of disenrollment than children with insured mothers at all three thresholds used to define coverage gaps. Particular combinations of maternal-child cash assistance receipt remained protective for any gap and gaps $>14$ days, but not for gaps $>60$ days. Consistent with single variable results, maternal knowledge that TANF and Medicaideligibility criteria differ and monthly household income remained associated with child disenrollment.

In the adjusted models analyzing time to the child's first Medicaid disenrollment, maternal disenrollment was associated with a more than 4 times increased rate of child disenrollment. Consistent with our findings using odds ratios, children receiving cash assistance and those whose families had higher household income demonstrated lower rates of disenrollment (Table 5).

\section{Discussion}

In this study population, maternal Medicaid enrollment status was significantly and strongly associated with child Medicaid enrollment status. This association between maternal disenrollment and child disenrollment remained strong and significant for gaps of any length and after adjusting for relevant covariates. Consistent with our hypotheses and Aday and Anderson's framework [8], maternal and child cash assistance receipt and maternal knowledge about differences in eligibility criteria for Medicaid and TANF were significantly associated with child Medicaid enrollment status. As expected, maternal disenrollment, household income, and cash assistance receipt are associated with time to child's first disenrollment. Our findings are consistent with 
Table 2 Odds ratios for predictors of child Medicaid disenrollment based on single predictor variable GEE models

\begin{tabular}{|c|c|c|c|c|c|c|}
\hline \multirow[t]{2}{*}{ Variable } & \multicolumn{2}{|l|}{ Any gap } & \multicolumn{2}{|l|}{$>14$ day gap } & \multicolumn{2}{|l|}{$>60$ day gap } \\
\hline & $\begin{array}{l}\text { Odds Ratio } \\
(95 \% \text { Cl) }\end{array}$ & $P$-value & $\begin{array}{l}\text { Odds Ratio } \\
(95 \% \mathrm{Cl})\end{array}$ & $P$-value & $\begin{array}{l}\text { Odds Ratio } \\
(95 \% \mathrm{Cl})\end{array}$ & $P$-value \\
\hline \multicolumn{7}{|l|}{ Maternal disenrollment } \\
\hline Under Medicaid coverage & Ref. & - & Ref. & - & Ref. & - \\
\hline Disenrolled from Medicaid (any gap) & $11.97(8.09,17.72)$ & $<0.001$ & $10.81(7.09,16.48)$ & $<0.001$ & $15.76(9.71,25.58)$ & $<0.001$ \\
\hline \multicolumn{7}{|l|}{ Child cash assistance status } \\
\hline No & Ref. & - & Ref. & - & Ref. & - \\
\hline Yes & $0.29(0.20,0.41)$ & $<0.001$ & $0.33(0.23,0.48)$ & $<0.001$ & $0.29(0.19,0.45)$ & $<0.001$ \\
\hline \multicolumn{7}{|l|}{ Mother cash assistance status } \\
\hline No & Ref. & - & Ref. & - & Ref. & - \\
\hline Yes & $0.77(0.56,1.06)$ & 0.11 & $0.87(0.62,1.23)$ & 0.44 & $0.84(0.56,1.24)$ & 0.37 \\
\hline \multicolumn{7}{|l|}{ Combined cash assistance status } \\
\hline Neither had cash assistance & Ref. & - & Ref. & - & Ref. & - \\
\hline Only mother had cash assistance & $1.47(0.92,2.34)$ & 0.10 & $1.71(1.03,2.82)$ & 0.036 & $1.59(0.92,2.76)$ & 0.099 \\
\hline Only child had cash assistance & $0.30(0.18,0.52)$ & $<0.001$ & $0.37(0.21,0.65)$ & $<0.001$ & $0.30(0.15,0.58)$ & $<0.001$ \\
\hline Both had cash assistance & $0.32(0.21,0.49)$ & $<0.001$ & $0.39(0.25,0.60)$ & $<0.001$ & $0.35(0.21,0.58)$ & $<0.001$ \\
\hline \multicolumn{7}{|l|}{ Maternal age } \\
\hline & $0.99(0.95,1.02)$ & 0.41 & $0.99(0.96,1.03)$ & 0.66 & $0.99(0.95,1.03)$ & 0.61 \\
\hline \multicolumn{7}{|l|}{ Maternal race } \\
\hline African American & Ref. & - & Ref. & - & Ref. & - \\
\hline Other & $1.09(0.75,1.57)$ & 0.65 & $1.17(0.77,1.77)$ & 0.46 & $1.18(0.74,1.89)$ & 0.48 \\
\hline \multicolumn{7}{|l|}{ Marital status } \\
\hline Single/widowed/divorced & Ref. & - & Ref. & - & Ref. & - \\
\hline Married & $1.56(0.90,2.70)$ & 0.11 & $1.88(1.07,3.30)$ & 0.029 & $2.23(1.22,4.07)$ & 0.0090 \\
\hline \multicolumn{7}{|l|}{ Maternal health literacy ${ }^{*}$} \\
\hline Inadequate & Ref. & - & Ref. & - & Ref. & - \\
\hline Marginal & $0.71(0.38,1.33)$ & 0.29 & $0.79(0.40,1.60)$ & 0.52 & $0.74(0.31,1.73)$ & 0.48 \\
\hline Adequate & $0.79(0.49,1.29)$ & 0.35 & $0.76(0.43,1.34)$ & 0.34 & $0.87(0.44,1.71)$ & 0.66 \\
\hline \multicolumn{7}{|l|}{ Maternal education } \\
\hline Less than High School & Ref. & - & Ref. & - & Ref. & - \\
\hline High School & $1.08(0.71,1.66)$ & 0.70 & $1.11(0.69,1.76)$ & 0.66 & $1.01(0.59,1.71)$ & 0.97 \\
\hline More than High School & $1.10(0.76,1.59)$ & 0.60 & $1.08(0.72,1.62)$ & 0.72 & $0.96(0.61,1.53)$ & 0.87 \\
\hline \multicolumn{7}{|c|}{ Maternal knowledge that TANF and Medicaid eligibility criteria differ } \\
\hline Yes & Ref. & - & Ref. & - & Ref. & - \\
\hline No & $1.47(1.02,2.13)$ & 0.038 & $1.33(0.87,2.05)$ & 0.18 & $1.27(0.78,2.07)$ & 0.34 \\
\hline \multicolumn{7}{|l|}{ Maternal self-reported health ${ }^{*}$} \\
\hline Total $<80$ (poor health) & Ref. & - & Ref. & - & Ref. & - \\
\hline Total $\geq 80$ (good health) & $0.95(0.67,1.34)$ & 0.76 & $0.94(0.63,1.40)$ & 0.76 & $0.74(0.61,1.44)$ & 0.78 \\
\hline \multicolumn{7}{|l|}{ Prenatal care, self-reported ${ }^{*}$} \\
\hline All/Most of the time & Ref. & - & Ref. & - & Ref. & - \\
\hline Some or none of the time & $0.81(0.46,1.41)$ & 0.45 & $0.79(0.44,1.44)$ & 0.44 & $0.70(0.33,1.50)$ & 0.36 \\
\hline \multicolumn{7}{|l|}{ Maternal social support ${ }^{*}$} \\
\hline Low & Ref. & - & Ref. & - & Ref. & - \\
\hline Medium & $0.74(0.46,1.18)$ & 0.20 & $0.85(0.51,1.41)$ & 0.53 & $1.04(0.52,2.08)$ & 0.92 \\
\hline High & $1.06(0.69,1.62)$ & 0.80 & $1.35(0.83,2.20)$ & 0.23 & $1.91(1.03,3.52)$ & 0.04 \\
\hline Not collected & $0.37(0.21,0.65)$ & 0.0006 & $0.33(0.16,0.68)$ & 0.003 & $0.14(0.04,0.51)$ & 0.003 \\
\hline \multicolumn{7}{|l|}{ Household income ${ }^{*}$} \\
\hline$<\$ 1000 /$ month & Ref. & - & Ref. & - & Ref. & - \\
\hline$\$ 1000$ or more /month & $1.05(0.74,1.50)$ & 0.78 & $1.00(0.68,1.46)$ & 0.99 & $1.39(0.90,2.16)$ & 0.14 \\
\hline
\end{tabular}

Maternal employment status* 
Table 2 Odds ratios for predictors of child Medicaid disenrollment based on single predictor variable GEE models (Continued)

\begin{tabular}{|c|c|c|c|c|c|c|}
\hline \multirow[t]{2}{*}{ Variable } & \multicolumn{2}{|l|}{ Any gap } & \multicolumn{2}{|l|}{$>14$ day gap } & \multicolumn{2}{|l|}{$>60$ day gap } \\
\hline & $\begin{array}{l}\text { Odds Ratio } \\
(95 \% \text { Cl) }\end{array}$ & $P$-value & $\begin{array}{l}\text { Odds Ratio } \\
(95 \% \text { CI) }\end{array}$ & $P$-value & $\begin{array}{l}\text { Odds Ratio } \\
(95 \% \mathrm{Cl})\end{array}$ & $P$-value \\
\hline Student & Ref. & - & Ref. & - & Ref. & - \\
\hline Employed & $1.33(0.89,1.98)$ & 0.16 & $1.42(0.92,2.20)$ & 0.12 & $1.18(0.71,1.96)$ & 0.52 \\
\hline Unemployed & $0.95(0.62,1.47)$ & 0.83 & $1.06(0.66,1.70)$ & 0.81 & $0.85(0.50,1.96)$ & 0.55 \\
\hline \multicolumn{7}{|l|}{ Other children in household } \\
\hline None & Ref. & - & Ref. & - & Ref. & - \\
\hline One & $0.76(0.51,1.14)$ & 0.18 & $0.90(0.58,1.40)$ & 0.64 & $0.64(0.38,1.09)$ & 0.098 \\
\hline Two or more & $0.72(0.50,1.05)$ & 0.089 & $0.86(0.57,1.30)$ & 0.47 & $0.88(0.55,1.40)$ & 0.59 \\
\hline \multicolumn{7}{|l|}{ Family housing situation ${ }^{*}$} \\
\hline Lives in own housing & Ref. & - & Ref. & - & Ref. & - \\
\hline Rents or lives with relatives/friends & $1.42(1.01,1.99)$ & 0.04 & $1.34(0.93,1.93)$ & 0.11 & $1.43(0.94,2.17)$ & 0.09 \\
\hline \multicolumn{7}{|l|}{ Travel time to Medicaid office } \\
\hline$\leq 30 \mathrm{~min}$ & Ref. & - & Ref. & - & Ref. & - \\
\hline$>30 \mathrm{~min}$ & $1.01(0.70,1.46)$ & 0.97 & $1.16(0.79,1.70)$ & 0.44 & $1.17(0.73,1.86)$ & 0.50 \\
\hline
\end{tabular}

other studies $[11,15]$ that together underscore the importance of supporting family coverage and continued outreach efforts to potential eligible populations in order to improve child Medicaid retention.

We found that a greater proportion of mothers in the cohort experienced disenrollment than children. The higher rate of unstable Medicaid coverage for mothers may be related to a more burdensome application process and/or differences in income eligibility thresholds for adults than for children. During 2005-2006, there were only 27 states that had family-friendly applications where parents could complete a single application for their child and themselves [33]. While only six states required an asset test for child Medicaid applications, 30 states required asset tests for parent Medicaid applications [33]. In the wake of the 2010 Affordable Care Act (ACA) implementation, states have focused on further streamlining the Medicaid application and renewal processes by leveraging technology and using a single application for the entire family such that the children's uninsured rate reached a historic low of less than $5 \%$ [34]. Our findings indicate that repealing the ACA Medicaid expansion is likely to have adverse impact on child Medicaid enrollment. It is unclear whether policy makers will continue to support ACA expansions and streamlining Medicaid application processes in the long-term.

Mother-child cash assistance receipt and child cash assistance receipt had strong protective effects against child disenrollment. At the same time, about $20 \%$ of the mothers in this study did not know that Medicaid and TANF had separate eligibility processes. The TANF enrollment process is as complicated, if not more so, as the Medicaid enrollment process $[11,13]$. One plausible explanation for our finding is that parents who were able to navigate the cash assistance application process were also more likely to know how to navigate the Medicaid application process, thus lowering the likelihood of the child's disenrollment from Medicaid. Since the ACA was implemented, states have improved outreach efforts to assist eligible parents and children to enroll in Medicaid. Most states now offer web-based accounts to manage Medicaid coverage after enrollment and more than half have a portal that enables consumer assisters to submit applications on behalf of individuals that they help [35]. Effective outreach and enrollment efforts will be needed to continue to reach eligible families, both old and new, to facilitate enrollment in expanded Medicaid programs.

We also found that the set of predictors significantly associated with child Medicaid disenrollment changed when the threshold for defining gaps lengthened. Specifically, using > 60 days as the threshold for defining gaps resulted in family housing situation becoming a significant predictor whereas household monthly income and maternal knowledge that Medicaid and TANF have different eligibility criteria did not remain significant. This change in predictors suggests that families whose children had longer gaps face different barriers to Medicaid renewal than families whose children had shorter gaps. These findings are consistent with results from other states $[4,16]$ and suggest different outreach and assistance efforts - such as targeted assistance to maintain coverage for families in unstable housing--- are needed when trying to reach families of children with different lengths of coverage gaps.

There are some limitations to this study. First, child Medicaid enrollment patterns were only observed for its first 24 months of life. As children grow older and family 
Table 3 Hazard ratios for predictors of child's time to first Medicaid disenrollment based on single predictor variable Cox proportional hazard models

\begin{tabular}{|c|c|c|}
\hline \multirow[t]{2}{*}{ Variable } & \multicolumn{2}{|c|}{ Time to First Disenrollment } \\
\hline & $\begin{array}{l}\text { Hazard Ratio } \\
(95 \% \mathrm{Cl})\end{array}$ & P-value \\
\hline \multicolumn{3}{|l|}{ Maternal disenrollment } \\
\hline Under Medicaid coverage & Ref. & - \\
\hline Disenrolled from Medicaid (any gap) & $5.48(4.02,7.46)$ & $<0.001$ \\
\hline \multicolumn{3}{|l|}{ Child cash assistance status } \\
\hline No & Ref. & - \\
\hline Yes & $0.31(0.23,0.42)$ & $<0.001$ \\
\hline \multicolumn{3}{|l|}{ Mother cash assistance status } \\
\hline No & Ref. & - \\
\hline Yes & $0.86(0.66,1.13)$ & 0.29 \\
\hline \multicolumn{3}{|l|}{ Combined cash assistance status } \\
\hline Neither had cash assistance & Ref. & - \\
\hline Only mother had cash assistance & $1.32(0.95,1.84)$ & 0.10 \\
\hline Only child had cash assistance & $0.31(0.20,0.48)$ & $<0.001$ \\
\hline Both had cash assistance & $0.36(0.24,0.52)$ & $<0.001$ \\
\hline \multicolumn{3}{|l|}{ Maternal age } \\
\hline & $0.98(0.96,1.01)$ & 0.19 \\
\hline \multicolumn{3}{|l|}{ Maternal race } \\
\hline African American & Ref. & - \\
\hline Other & $1.33(0.96,1.84)$ & 0.09 \\
\hline \multicolumn{3}{|l|}{ Marital status } \\
\hline Single/widowed/divorced & Ref. & - \\
\hline Married & $1.28(0.88,1.86)$ & 0.20 \\
\hline \multicolumn{3}{|l|}{ Maternal health literacy ${ }^{*}$} \\
\hline Inadequate & Ref. & - \\
\hline Marginal & $0.74(0.48,1.13)$ & 0.16 \\
\hline Adequate & $0.66(0.38,1.14)$ & 0.13 \\
\hline \multicolumn{3}{|l|}{ Maternal education } \\
\hline Less than High School & Ref. & - \\
\hline High School & $0.97(0.67,1.40)$ & 0.88 \\
\hline More than High School & $0.90(0.63,1.21)$ & 0.48 \\
\hline \multicolumn{3}{|c|}{ Maternal knowledge that TANF and Medicaid eligibility criteria differ } \\
\hline Yes & Ref. & - \\
\hline No & $1.35(0.98,1.85)$ & 0.07 \\
\hline \multicolumn{3}{|l|}{ Maternal self-reported health ${ }^{*}$} \\
\hline Total < 80 (poor health) & Ref. & - \\
\hline Total $\geq 80$ (good health) & $0.85(0.64,1.13)$ & 0.26 \\
\hline \multicolumn{3}{|l|}{ Prenatal care ${ }^{*}$} \\
\hline All/Most of the time & Ref. & - \\
\hline Some or none of the time & $1.04(0.62,1.72)$ & 0.89 \\
\hline \multicolumn{3}{|l|}{ Maternal social support ${ }^{*}$} \\
\hline Low & Ref. & - \\
\hline Medium & $0.78(0.51,1.21)$ & 0.27 \\
\hline High & $1.10(0.78,1.57)$ & 0.58 \\
\hline Not collected & $1.47(0.86,2.52)$ & 0.16 \\
\hline
\end{tabular}

Table 3 Hazard ratios for predictors of child's time to first Medicaid disenrollment based on single predictor variable Cox proportional hazard models (Continued)

\begin{tabular}{|c|c|c|}
\hline \multirow[t]{2}{*}{ Variable } & \multicolumn{2}{|c|}{ Time to First Disenrollment } \\
\hline & $\begin{array}{l}\text { Hazard Ratio } \\
(95 \% \text { Cl) }\end{array}$ & P-value \\
\hline \multicolumn{3}{|l|}{ Household income ${ }^{*}$} \\
\hline$<\$ 1000 /$ month & Ref. & - \\
\hline$\$ 1000$ or more /month & $0.91(0.68,1.22)$ & 0.53 \\
\hline \multicolumn{3}{|l|}{ Maternal employment status ${ }^{*}$} \\
\hline Student & Ref. & - \\
\hline Employed & $0.91(0.65,1.29)$ & 0.92 \\
\hline Unemployed & $0.80(0.55,1.18)$ & 0.80 \\
\hline \multicolumn{3}{|l|}{ Other children in household } \\
\hline None & Ref. & - \\
\hline One & $0.59(0.42,0.82)$ & 0.002 \\
\hline Two or more & $0.64(0.47,0.87)$ & 0.005 \\
\hline \multicolumn{3}{|l|}{ Family housing situation ${ }^{*}$} \\
\hline Lives in own housing & Ref. & - \\
\hline Rents or lives with relatives/friends & $1.64(1.26,2.14)$ & 0.0003 \\
\hline \multicolumn{3}{|l|}{ Travel time to Medicaid office ${ }^{*}$} \\
\hline$\leq 30 \min$ & Ref. & - \\
\hline$>30 \mathrm{~min}$ & $1.01(0.74,1.37)$ & 0.96 \\
\hline
\end{tabular}

Note: Maternal disenrollment was defined as having any gap in Medicaid coverage. Maternal health literacy was assessed using the S-TOFHLA and categorized as inadequate, marginal, or adequate per published technical guidance (Nurss JR, Parker R, Willams M, Baker D. TOFHLA: Test of Functional Health Literacy in Adults. Second ed. Snow Camp, NC: Peppercorn Books \& Press; 2001). Maternal instrumental and relational social support was assessed using the Maternal Social Support Index and categorized low, medium, or high using tertiles per published technical guidance (Pascoe JM, lalongo NS, Horn WF, Reinhart MA, Perradatto D. The reliability and validity of the maternal social support index. Fam. Med. Jul-Aug 1988;20(4):271-27)

"Results from 10 imputed datasets

Entries in boldface have $p$-values less than 0.05

characteristics change, the relationship between child and maternal Medicaid enrollment patterns is also likely to weaken. Notably, in this study, we do not assess the types of disenrollment (e.g., increased household income, termination of emergency Medicaid, etc.) and maternal disenrollment is not a random event. However, from the perspective of patients and providers, nearly one-quarter of low-income adults still experience 'churning, (i.e. moving between and out of health plans) in the post-ACA era with adverse consequences including disrupted care and medication adherence, increased emergency department use, and worsening self-reported quality of care [36-38]. Second, this study cohort is primarily comprised of African-American families living in an urban area. Further studies among diverse populations are needed to assess generalizability of these findings. Third, we assessed maternal health literacy using only the S-TOFHLA and did not find a significant association between health literacy and child Medicaid disenrollment. A recent review of 19 health literacy indices 
Table 4 Odds ratios for predictors of child Medicaid disenrollment from best-fitting GEE models

\begin{tabular}{|c|c|c|c|c|c|c|c|c|c|}
\hline \multirow[t]{2}{*}{ Variable } & \multicolumn{3}{|c|}{ Any gap } & \multicolumn{3}{|c|}{ > 14-day gap } & \multicolumn{3}{|c|}{ >60-day gap } \\
\hline & $\begin{array}{l}\text { Odds } \\
\text { Ratio }\end{array}$ & $\begin{array}{l}95 \% \text { confidence } \\
\text { interval }\end{array}$ & $P$-value & $\begin{array}{l}\text { Odds } \\
\text { Ratio }\end{array}$ & $\begin{array}{l}95 \% \text { confidence } \\
\text { interval }\end{array}$ & $P$-value & $\begin{array}{l}\text { Odds } \\
\text { Ratio }\end{array}$ & $\begin{array}{l}95 \% \text { confidence } \\
\text { interval }\end{array}$ & $P$-value \\
\hline \multicolumn{10}{|l|}{ Maternal disenrollment } \\
\hline Under Medicaid coverage & Ref. & - & - & Ref. & - & - & Ref. & - & - \\
\hline $\begin{array}{l}\text { Disenrolled from Medicaid } \\
\text { (any gap) }\end{array}$ & 12.60 & $(8.11,19.58)$ & $<0.001$ & 11.78 & $(7.38,18.82)$ & $<0.001$ & 16.75 & $(9.67,29.02)$ & $<0.001$ \\
\hline \multicolumn{10}{|c|}{ Maternal knowledge that Medicaid and TANF eligibility criteria differ } \\
\hline Yes & Ref. & - & - & Ref. & - & - & - & - & - \\
\hline No & 2.01 & $(1.21,3.35)$ & 0.007 & 1.81 & $(1.05,3.13)$ & 0.03 & - & - & - \\
\hline \multicolumn{10}{|l|}{ Combined cash assistance status } \\
\hline Neither had cash assistance & Ref. & - & - & Ref. & - & - & Ref. & - & - \\
\hline $\begin{array}{l}\text { Only mother had cash } \\
\text { assistance }\end{array}$ & 1.85 & $(0.99,3.41)$ & 0.05 & 2.11 & $(1.11,3.99)$ & 0.02 & 2.15 & $(0.95,4.86)$ & 0.07 \\
\hline $\begin{array}{l}\text { Only child had cash } \\
\text { assistance }\end{array}$ & 0.38 & $(0.19,0.76)$ & 0.006 & 0.48 & $(0.23,0.98)$ & 0.04 & 0.50 & $(0.22,1.14)$ & 0.10 \\
\hline Both had cash assistance & 0.48 & $(0.29,0.82)$ & 0.007 & 0.59 & $(0.34,1.04)$ & 0.07 & 0.78 & $(0.41,1.46)$ & 0.43 \\
\hline \multicolumn{10}{|l|}{ Household income ${ }^{*}$} \\
\hline$<\$ 1000 /$ month & Ref. & - & - & Ref. & - & - & - & - & - \\
\hline$\$ 1000$ or more /month & 0.59 & $(0.36,0.96)$ & 0.03 & 0.57 & $(0.34,0.94)$ & 0.03 & - & - & - \\
\hline \multicolumn{10}{|l|}{ Family housing situation $^{*}$} \\
\hline Lives in own housing & - & - & - & - & - & - & Ref. & & - \\
\hline $\begin{array}{l}\text { Rents or lives with } \\
\text { relatives/friends }\end{array}$ & - & - & - & - & - & - & 2.08 & $(1.21,3.56)$ & 0.008 \\
\hline
\end{tabular}

Note: Maternal disenrollment was defined as having any gap in Medicaid coverage. All models were based on 604 dyads. The best fitting model was selected based on the QIC and the QICu. All final models were checked to ensure that adding another variable did not significantly change the QIC or the QICu ${ }^{*}$ Results from 10 imputed datasets

Entries in boldface have $p$-values less than 0.05

Table 5 Hazard ratio for child's time to first disenrollment

\begin{tabular}{|c|c|c|c|}
\hline \multirow[t]{2}{*}{ Variable } & \multicolumn{3}{|c|}{ Time to first disenrollment } \\
\hline & $\begin{array}{l}\text { Hazard } \\
\text { Ratio }\end{array}$ & $\begin{array}{l}95 \% \text { confidence } \\
\text { interval }\end{array}$ & P-value \\
\hline \multicolumn{4}{|l|}{ Maternal disenrollment } \\
\hline Under Medicaid coverage & Ref. & - & - \\
\hline $\begin{array}{l}\text { Disenrolled from Medicaid } \\
\text { (any gap) }\end{array}$ & 4.80 & $(3.48,6.61)$ & $<0.001$ \\
\hline \multicolumn{4}{|l|}{ Household income* } \\
\hline$<\$ 1000 /$ month & Ref. & - & - \\
\hline$\$ 1000$ or more /month & 0.62 & $(0.46,0.83)$ & 0.0016 \\
\hline \multicolumn{4}{|c|}{ Combined cash assistance status } \\
\hline $\begin{array}{l}\text { Neither had cash } \\
\text { assistance }\end{array}$ & Ref. & - & - \\
\hline $\begin{array}{l}\text { Only mother had cash } \\
\text { assistance }\end{array}$ & 1.28 & $(0.92,1.78)$ & 0.15 \\
\hline $\begin{array}{l}\text { Only child had cash } \\
\text { assistance }\end{array}$ & 0.38 & $(0.24,0.59)$ & $<0.001$ \\
\hline Both had cash assistance & 0.52 & $(0.35,0.78)$ & 0.001 \\
\hline
\end{tabular}

Note: Maternal disenrollment was defined as having any gap in

Medicaid coverage

"Results from 10 imputed datasets

Entries in boldface have $p$-values less than 0.05 concluded that none of the currently available health literacy measures fully assesses a person's ability to obtain, process, and understand health information, however the TOFHLA demonstrates the strongest psychometric properties of all the instruments examined [39].

\section{Conclusions}

We found that maternal Medicaid disenrollment is associated with a more than 10 times increased odds of child Medicaid disenrollment, regardless of the duration of the gap. Children who experienced shorter gaps in coverage faced some different barriers than children who experienced longer gaps in coverage. With ACA currently in effect, many more new families are eligible for publicly funded health insurance, in addition to those eligible families who were not previously enrolled. To ensure all eligible families can take advantage of these coverage opportunities, policymakers need to invest in effective and appropriate outreach strategies and provide family-friendly application processes to reduce enrollment barriers. 


\section{Appendix 1}

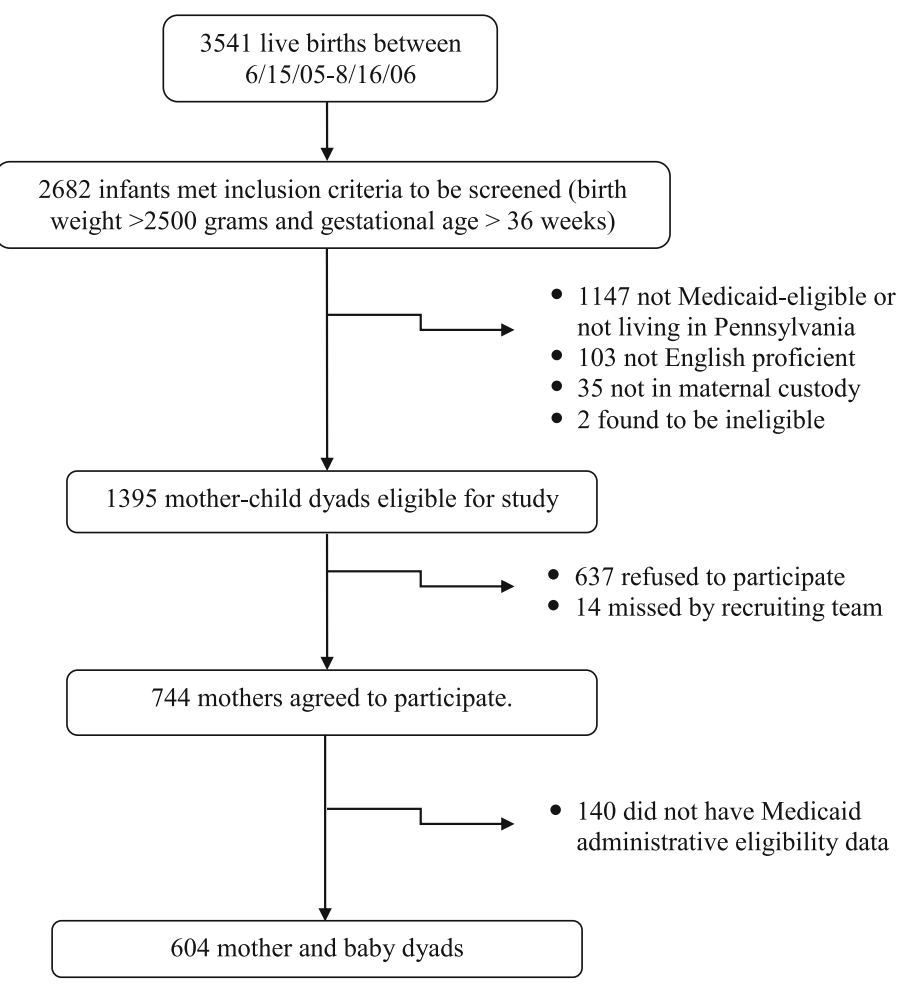

Authors: Susmita Pati, MD MPH, Rose Calixte, PhD, Angie Wong, MHS, Jiayu Huang PhD, Zeinab Baba, DrPh, Xianqun Luan, MS, Avital Cnaan, PhD

Fig. 2 Study enrollment protocol

\section{Appendix 2}

Table 6 Characteristics of children with and without Medicaid administrative eligibility data

\begin{tabular}{lll}
\hline & $\begin{array}{l}\text { Have Medicaid Data } \\
(N=604) \\
N(\%)\end{array}$ & $\begin{array}{l}\text { No Medicaid Data } \\
(N=140) \\
N(\%)\end{array}$ \\
\hline Mean Maternal Age (SD) & $23.2(5.2)$ & $24.8(5.8)$ \\
Maternal Race & $569(94)$ & $137(98)$ \\
African American & $35(6)$ & $3(2)$ \\
Other & & \\
Other children & $228(38)$ & $43(31)$ \\
None & $157(26)$ & $41(29)$ \\
One & $219(36)$ & $56(40)$ \\
Two or more & 0 & 2 \\
Missing & & \\
Education & $198(33)$ & $45(32)$ \\
Less than high school & $147(24)$ & $35(25)$ \\
High school & $259(43)$ & $60(43)$ \\
More than high school & & \\
Health Literacy & $54(9)$ & $15(11)$ \\
Inadequate & & 0.0035
\end{tabular}


Table 6 Characteristics of children with and without Medicaid administrative eligibility data (Continued)

\begin{tabular}{|c|c|c|c|}
\hline & $\begin{array}{l}\text { Have Medicaid Data } \\
(N=604) \\
\mathrm{N}(\%)\end{array}$ & $\begin{array}{l}\text { No Medicaid Data } \\
(N=140) \\
\mathrm{N}(\%)\end{array}$ & $P$-value \\
\hline Marginal & $77(13)$ & $12(9)$ & \\
\hline Adequate & $449(77)$ & 109(80) & \\
\hline Missing & 24 & 4 & \\
\hline \multicolumn{4}{|l|}{ Prenatal Care } \\
\hline All/Most of the time & $552(92)$ & $128(91)$ & 0.96 \\
\hline Some/None of the time & $51(8)$ & $12(9)$ & \\
\hline Missing & 1 & 0 & \\
\hline \multicolumn{4}{|l|}{ Income } \\
\hline$<\$ 1000 /$ month & $424(76)$ & 102(76) & 0.86 \\
\hline$\$ 1000$ or more/month & $132(24)$ & $33(24)$ & \\
\hline Missing & 48 & 5 & \\
\hline \multicolumn{4}{|l|}{ Marital Status } \\
\hline Single/Divorced/Widowed & $533(88)$ & 113(82) & 0.05 \\
\hline Married & $71(12)$ & $25(18)$ & \\
\hline Missing & 0 & 2 & \\
\hline \multicolumn{4}{|l|}{ Country } \\
\hline US Born & 573(95) & $112(80)$ & $<0.0001$ \\
\hline Non-US Born & $31(5)$ & $28(20)$ & \\
\hline \multicolumn{4}{|l|}{ Maternal Social Support Index } \\
\hline Low & $151(34)$ & $32(36)$ & 0.83 \\
\hline Medium & $148(33)$ & $31(35)$ & \\
\hline High & 144(33) & $26(29)$ & \\
\hline Not Applicable & 126 & 39 & \\
\hline Missing & 35 & 12 & \\
\hline
\end{tabular}

Note: P-value was for the exact $\mathrm{x}^{2}$ test of association for categorical variables. T-test was used for maternal age

\section{Appendix 3 Imputation Methods}

As with typical pattern of missingness of responses in surveys, many subjects failed to complete or respond properly to one or more item. The fraction of missing survey data ranged from $0.17 \%$ to $16.06 \%$ per item, with variables for maternal employment and knowledge that Medicaid and TANF eligibility criteria differ having more than $10 \%$ missing. For each of the chained equations, we selected those other variables that in theory would predict the unobserved values. This method requires that each variable with a missing value be specified in a regression equation with the proper form (e.g., nominal, binary, etc.) for the missing values. Continuous variables were imputed using a linear regression model, binary variables with missing values were imputed using a logistic regression, while those with nominal values used multinomial logit regression. Several variables were clearly ordered (e.g., education, income category) and were imputed using ordinal logistic regression. The chained equation methods imputes one value at a time, and then with the value filled in, proceeded to the next missing variable and its equation, which then assumed that the imputed value is known. At each imputation, the value chosen is taken from the posterior predictive distribution of from the regression, and unlike simple mean or regression-based imputation, this method adequately accounts for the additional variance arising when values must be filled in. The chain of equations is then repeated in 10 cycles to achieve better convergence. The entire process of 10 cycles is then repeated 10 times to obtain 10 sets of imputed values. For each of the chained equations, we selected variables that in theory would predict the unobserved values. In addition, as is proper for imputation, we included maternal age, child gender, and whether the child disenrolled at any time as auxiliary variables. This imputation permits the proper estimation of variance by combining two variance components: the average of the within imputation variances and the across imputation variance. ${ }^{32}$ All reported results are from completed datasets using the imputation procedures previously described and analyzed in $\mathrm{SAS}^{\circ} \mathrm{v} 9.1$.

\section{Abbreviations}

ACA: Affordable Care Act; AIC: Akaike information criterion; GEE: Generalized estimating equations; HIP: Health Insurance Improvement Project; MSSI: Maternal Social Support Index; OR: Odds ratio; S-TOFHLA: Short-Test of Functional Health Literacy in Adults; TANF: Temporary Assistance to Needy Families 


\section{Acknowledgements}

We gratefully acknowledge the contributions of Suraj K. Bhatt, Marlon Satchell, Jane Kavanagh, and the Health Insurance Improvement Project study team to this work. We thank the network of primary care physicians, their patients and families for their contribution to clinical research through the Pediatric Research Consortium (PeRC) at The Children's Hospital of Philadelphia. Medicaid administrative eligibility files were obtained from the Pennsylvania Department of Public Welfare through a data use agreement. The content is solely the responsibility of the authors and does not necessarily represent the official views of the Pennsylvania Department of Public Welfare.

\section{Funding}

Dr. Pati and Dr. Baba were partially supported by a K23 HD047655 from the National Institute of Child Health and Human Development and The Children's Hospital of Philadelphia Foerderer-Murray Award. Dr. Cnaan was partially supported by Award Number UL1TR000075 from the NIH National Center for Advancing Translational Sciences. The contents of this manuscript are solely the responsibility of the authors and do not necessarily represent the official views of the National Center for Advancing Translational Sciences or the National Institutes of Health. The funding bodies had no role in the study design, collection, analysis, data interpretation, or manuscript writing.

\section{Availability of data and materials}

The survey used in this study is included with this article's additional file. The data used in this work will not be shared because these files contain individually identifiable information (e.g., Medicaid enrollment numbers and dates).

\section{Authors' contributions}

SP conceived and designed the study, reviewed and interpreted study results, and led the manuscript writing. $\mathrm{RC}, J \mathrm{H}, \mathrm{ZB}$, and $\mathrm{XL}$ performed statistical analyses and assisted in writing the manuscript. AW made critical revisions to the manuscript. AC helped design the study, led the statistical analyses, and assisted in writing the manuscript. All authors read and approved the final manuscript

\section{Authors' information}

SP is Professor and Chief, Division of Primary Care Pediatrics at Stony Brook University. AC is Professor of Pediatrics at Georgetown University, Children's National Medical Center.

\section{Ethics approval and consent to participate}

This study was approved by and carried out in accordance with guidelines from the Institutional Review Boards at the University of Pennsylvania (IRB\# 802413), The Children's Hospital of Philadelphia (IRB\# 07-004091), and Stony Brook University (IRB \# 2010-1250). All subjects included in this study provided informed consent (i.e. written informed consent from study participants and, for children, written informed consent from the parent/guardian for the child) in accordance with guidelines from the aforementioned Institutional Review Boards.

\section{Consent for publication}

Not applicable.

\section{Competing interests}

The authors declare that they have no competing interests.

\section{Publisher's Note}

Springer Nature remains neutral with regard to jurisdictional claims in published maps and institutional affiliations.

\section{Author details}

${ }^{1}$ Division of Primary Care Pediatrics, State University of New York at Stony Brook, 100 Nicolls Rd, Stony Brook, NY 11794, USA. ²Pediatric Generalist Research Group, The Children's Hospital of Philadelphia, 3401 Civic Center Blvd, Philadelphia, PA 19104, USA. 'Healthcare Analytics Unit, The Children's Hospital of Philadelphia, 3401 Civic Center Blvd, Philadelphia, PA 19104, USA. ${ }^{4}$ School of Medicine and Health Sciences, The George Washington University, 2121 I St NW, Washington, DC 20052, USA. ${ }^{5}$ Center for Clinical and Translational Science, Children's National Medical Center, 111 Michigan Ave NW, Washington, DC 20010, USA.
Received: 4 January 2016 Accepted: 2 August 2018

Published online: 21 August 2018

\section{References}

1. Olson LM, Tang SF, Newacheck PW. Children in the United States with discontinuous health insurance coverage. N Engl J Med. 2005;353(4):382-91.

2. Cassedy A, Fairbrother G, Newacheck PW. The impact of insurance instability on children's access, utilization, and satisfaction with health care. Ambul Pediatr. 2008;8(5):321-8.

3. Cummings JR, Lavarreda SA, Rice T, Brown ER. The effects of varying periods of uninsurance on children's access to health care. Pediatrics. 2009:123(3):e411-8.

4. DeVoe JE, Graham A, Krois L, Smith J, Fairbrother GL. "Mind the gap" in children's health insurance coverage: does the length of a child's coverage gap matter. Ambul Pediatr. 2008;8(2):129-34.

5. Ku L, McTaggart P, Pervez F, Rosenbaum S. Improving Medicaid's continuity of coverage and quality of care. Washington D.C.: George Washington University; 2009

6. Kaiser Commission on Medicaid and the Uninsured. Medicaid eligibility, enrollment simplification, and coordination under the Affordable Care Act: A Summary of CMS's August 17, 2011. In: Proposed rule and key issues to consider. Washington, DC: The Henry J. Kaiser Family Foundation; 2011.

7. Health Insurance Coverage of Children 0-18. State Health Facts http://kff. org/other/state-indicator/children-0-18/. Accessed 8 Aug 2018.

8. Aday LA, Andersen RM. Equity of access to medical care: a conceptual and empirical overview. Med Care. 1981;19(12):4-27.

9. Federico SG, Steiner JF, Beaty B, Crane L, Kempe A. Disruptions in insurance coverage: patterns and relationship to health care access, unmet need, and utilization before enrollment in the state Children's health insurance program. Pediatrics. 2007;120(4):e1009-16.

10. Bethell CD, Kogan MD, Strickland BB, Schor EL, Robertson J, Newacheck PW. A national and state profile of leading health problems and health care quality for US children: key insurance disparities and across-state variations. Acad Pediatr. 2011;11(3 Suppl):S22-33.

11. DeVoe JE, Marino M, Angier $\mathrm{H}$, et al. Effect of expanding Medicaid for parents on children's health insurance coverage: lessons from the Oregon experiment. JAMA Pediatr. 2015;169(1):e143145.

12. Pati S, Romero D, Chavkin W. Changes in use of health insurance and food assistance programs in medically underserved communities in the era of welfare reform: an urban study. Am J Public Health. 2002;92(9):1441-5.

13. U.S. General Accounting Office. Medicaid enrollment: Amid declines, state efforts to ensure coverage after Welfare Reform. Washington, D.C: U.S. General Accounting Office; 1999. https://www.gao.gov/products/162821.

14. Pati S, Kavanagh JE, Bhatt SK, Wong AT, Noonan K, Cnaan A. Reading level of Medicaid renewal applications. Acad Pediatr. 2012:12(4):297-301.

15. Sommers BD. Insuring children or insuring families: do parental and sibling coverage lead to improved retention of children in Medicaid and CHIP? J Health Econ. 2006;25(6):1154-69.

16. Yamauchi M, Carlson MJ, Wright BJ, Angier H, DeVoe JE. Does health insurance continuity among low-income adults impact their children's insurance coverage? Matern Child Health J. 2013;17(2):248-55.

17. DeVoe JE, Crawford C, Angier H, et al. The Association between Medicaid coverage for children and parents persists: 2002-2010. Matern Child Health J. 2015;19(8):1766-74.

18. Pati S, Mohamad Z, Cnaan A, Kavanagh J, Shea JA. Influence of maternal health literacy on child participation in social welfare programs: the Philadelphia experience. Am J Public Health. 2010;100(9):1662-5.

19. Nurss JR, Parker R, Willams M, Baker D. TOFHLA: test of functional health literacy in adults. Second ed. Snow Camp: Peppercorn Books \& Press; 2001.

20. Parker RM, Baker DW, Williams MV, Nurss JR. The test of functional health literacy in adults: a new instrument for measuring patients' literacy skills. J Gen Intern Med. 1995;10(10):537-41

21. Geoffrey RS, Cynthia B, Graham AB 3rd, et al. 2014 recommendations for pediatric preventive health care. Pediatrics. 2014;133(3):568-70.

22. US Department of Health and Human Services. Applying for Medicaid. https://longtermcare.acl.gov/medicare-medicaid-more/medicaid/applyingfor-medicaid.html. Accessed 8 Aug 2018.

23. Kogan MD, Newacheck PW, Blumberg SJ, et al. Underinsurance among children in the United States. N Engl J Med. 2010;363(9):841-51.

24. Flores G, Tomany-Korman SC. Racial and ethnic disparities in medical and dental health, access to care, and use of services in US children. Pediatrics. 2008;121(2):e286-98. 
25. Sommers BD. From Medicaid to uninsured: drop-out among children in public insurance programs. Health Serv Res. 2005;40(1):59-78.

26. Flores $G$, Bauchner H, Feinstein AR, Nguyen US. The impact of ethnicity, family income, and parental education on children's health and use of health services. Am J Public Health. 1999;89(7):1066-71.

27. Centers for Disease Control and Prevention. National Health Interview Survey. http://www.cdc.gov/nchs/nhis.htm. Accessed 8 Aug 2018.

28. Pascoe JM, lalongo NS, Horn WF, Reinhart MA, Perradatto D. The reliability and validity of the maternal social support index. Fam Med. 1988;20(4):271-6.

29. Hosmer DW, Lemeshow S. Applied logistic regression. Second ed: Online. New York: Wiley, Inc.; 2005

30. Pan W. Akaike's information criterion in generalized estimating equations. Biometrics. 2001;57(1):120-5.

31. Cantoni E, Flemming JM, Ronchetti E. Variable selection for marginal longitudinal generalized linear models. Biometrics. 2005;61(2):507-14.

32. van Buuren S. Multiple imputation of discrete and continuous data by fully conditional specification. Stat Methods Med Res. 2007:16(3):219-42.

33. Kaiser Commission on Medicaid and the Uninsured. Resuming the path to health coverage for children and parents: A 50 state update on eligibility rules, enrollment and renewal procedures, and cost-sharing practices in Medicaid and SCHIP in 2006. Washington, DC: The Henry J. Kaiser Family Foundation; 2007.

34. Ward BW, Clarke TC, Nugent CN, Schiller JS. Early release of selected estimates based on data from the 2015 National Health Interview Survey: Division of Health Interview Statistics, National Center for Health Statistics, Centers for Disease Control, U.S. Department of Health and Human Services; 2017. https:// www.cdc.gov/nchs/fastats/health-insurance.htm. Accessed 8 Aug 2018.

35. Kaiser Commission on Medicaid and the Uninsured. Medicaid and CHIP eligibility enrollment, renewal, and cost-sharing policies as of January 2017: Findings from a 50-state survey 2017. Washington, DC: The Henry J. Kaiser Family Foundation; 2017

36. Sommers BD, Gourevitch R, Maylone B, Blendon RJ, Epstein AM. Insurance churning rates for low-income adults under health reform: Lower than expected but still harmful for many. Health Aff. (Millwood). 2016;35(10): 1816-24.

37. Sommers BD, Rosenbaum S. Issues in health reform: how changes in eligibility may move millions back and forth between Medicaid and insurance exchanges. Health Aff. (Millwood). 2011;30(2):228-36.

38. Sommers BD, Graves JA, Swartz K, Rosenbaum S. Medicaid and marketplace eligibility changes will occur often in all states; policy options can ease impact. Health Aff (Millwood). 2014;33(4):700-7.

39. Jordan JE, Osborne RH, Buchbinder R. Critical appraisal of health literacy indices revealed variable underlying constructs, narrow content and psychometric weaknesses. J Clin Epidemiol. Apr 2011;64(4):366-79.

Ready to submit your research? Choose BMC and benefit from:

- fast, convenient online submission

- thorough peer review by experienced researchers in your field

- rapid publication on acceptance

- support for research data, including large and complex data types

- gold Open Access which fosters wider collaboration and increased citations

- maximum visibility for your research: over $100 \mathrm{M}$ website views per year

At BMC, research is always in progress.

Learn more biomedcentral.com/submissions 